\title{
The Graph of e-Abacus Diagram
}

\author{
AHMED MOHAMMED ALI ${ }^{1 *}$, AMMAR SEDDIQ MAHMOOD ${ }^{2}$ \\ ${ }^{1}$ Department of Mathematics, College of Computer science and Mathematics, University of Mosul, \\ Mosul, IRAQ \\ ${ }^{2}$ Department of Mathematics, College of Education for Pure science, University of Mosul, Mosul, \\ IRAQ
}

\begin{abstract}
Since James introduced the idea of an e-abacus diagram in 1978, this topic plays important roles with many topics, especially in algebra. In this paper, we will present new insights on this topic so that it appears to us as a graph theory; specifically a directed graph, according to certain conditions, to be a new start with another field. This proposed technique will be applied to the model that was approved by Mahmood and Mahmood in 2019 on English letters and to see how successful we are on Letter-Word-Sentence. It is completely natural that this method will have a new and very difficult method as an entrance to a new type of encryption process.
\end{abstract}

Key-Words: - directed graph, adjacent matrix, partition theory, e-abacus diagram.

Received: March 2, 2020. Revised: September 30, 2020. Accepted: October 13, 2020. Published: October 26, 2020.

\section{Introduction}

Let $r$ be a nonnegative integer. The composition $\pi=$ $\left(\pi_{1}, \pi_{2}, \ldots, \pi_{n}\right)=\left(\theta_{1}^{\gamma_{1}}, \theta_{2}^{\gamma_{2}}, \ldots, \theta_{m}^{\gamma_{m}}\right)$ (where $\gamma_{z}$ the number of times $\theta_{z}$ appeared, $\left.z=1,2, \ldots, m\right)$ of $r$ is a sequence of non-negative integers such that $|\pi|=\sum_{j=1}^{n} \pi_{j}=\sum_{z=1}^{m} \theta_{z}^{\gamma_{z}}=r$. The composition is called a partition of $r$ if $\pi_{j} \geq \pi_{j+1}, \forall j \geq 1$. Fix $\pi$ is a partition of $r$ and defining $\beta_{i}=\pi_{i}+b-i$, $1 \leq i \leq b$. The set $\left\{\beta_{1}, \beta_{2}, \cdots, \beta_{b}\right\}$ is said to be the set of $\beta$ - number for $\pi$, see [10]. Let $e$ be a positive integer number greater than or equal to 2 , we can represent numbers by a diagram called $e$-abacus diagram, see[5], as shown:

\section{Table 1.}

$e$-Abacus Diagram

\begin{tabular}{|c||c|c|c|}
\hline Runner 1 & Runner 2 & $\cdots$ & Runner $e$ \\
\hline 0 & 1 & $\cdots$ & $e-1$ \\
\hline \hline$e$ & $e+1$ & $\cdots$ & $2 e-1$ \\
\hline \hline $2 e$ & $2 e+1$ & $\cdots$ & $3 e-1$ \\
\hline \hline & $\vdots$ & $\cdots$ & $\vdots$ \\
\hline
\end{tabular}

where every $\beta$ will be represented by a $(0)$ and the rest of the sites by (-). In fact, the definition of $e$ Abacus diagram will leads us to the fact that the presence of an infinite number of diagrams are all suitable for any partition according to the value of $e$. Each partition will be represented first through by Young tableau $[\pi]$; see $[4]$, and later by e-Abacus diagram, for example, if $\pi=\left(3^{3}, 2,1^{2}\right)$ and $e=3$ or 4 , there exist many of $e$-abacus diagrams:

Table 2.

Young Tableau and $e$-Abacus Diagram of $\left(3^{3}, 2,1^{2}\right)$

\begin{tabular}{|c|c|c|c|c|c|c|c|c|c|c|c|c|c|c|}
\hline Young Tableau & \multicolumn{3}{|c|}{$e=3$} & \multicolumn{3}{|c|}{$e=3$} & \multicolumn{4}{|c|}{$e=4$} & \multicolumn{4}{|c|}{$e=4$} \\
\hline & • & $\bullet$ & - & - & $\bullet$ & - & - & - & $\bullet$ & - & - & $\bullet$ & $\bullet$ & $\bullet$ \\
\hline & $\bullet$ & $\bullet$ & - & - & $\bullet$ & - & - & $\bullet$ & - & $\bullet$ & - & $\bullet$ & $\bullet$ & - \\
\hline & - & - & $\bullet$ & - & - & - & $\bullet$ & - & - & - & - & - & - & $\bullet$ \\
\hline & - & - & - & & & & & & & & - & & - & - \\
\hline
\end{tabular}

Now, Many branches of mathematics, such as group theory, set theory, matrix theory and topology, have close connections with graph theory, therefore consider graph theory is one of the most important topics in applied sciences and engineering for because it is a mathematical model through which to analyse many concrete real-world problems successfully,[1]. There are two types of graphs: undirected graph is called graph and directed graph is called digraph. The graphs that we will be dealing with is directed graphs in this paper such that digraphs arise in a natural way in many applications of graph theory [3,6,11]. A graph $G$ is an ordered pair of non-empty set $V(G)$ of elements called vertices and a family $E(G)$ (it may be empty) from unordered pairs of vertices, each element of $E(G)$ is called an edge.A digraph $D$ as a set $V(D)$ that is non-empty of elements called vertices with a family $A(D)$ of ordered pairs of vertices is called each ordered pair of vertices $(u, v)$, where 
$u, v \in V(D)$ a directed edge ( directed edge from $u$ to $v)$. A directed graph is expressed as an ordered pair $(V(D), A(D))$. The number of vertex set $V(D)$ is the order digraph and the number of directed edges in $A(D)$ is the size digraph. A digraph $D$ is called finite if both $V(D)$ and $A(D)$ are finite [2]. In this paper, we will connecting between e-abacus diagram and directed graph by designing a specific matrix as a real input for this connection.

\section{2 e-Abacus Directed Graph}

By looking at any e-Abacus diagram, we will always see the existence of $(\bullet)$ and $(-)$, so if we assume that any (0) corresponds to 1 , any ( - ) corresponds to 0 and that this diagram is a matrix of elements 1 and 0 , thus becoming matrix for this diagram, (this matrix we call it e-abacus diagram representative matrix).By above example:

Table 3

The Matrix of $e$-Abacus Diagram

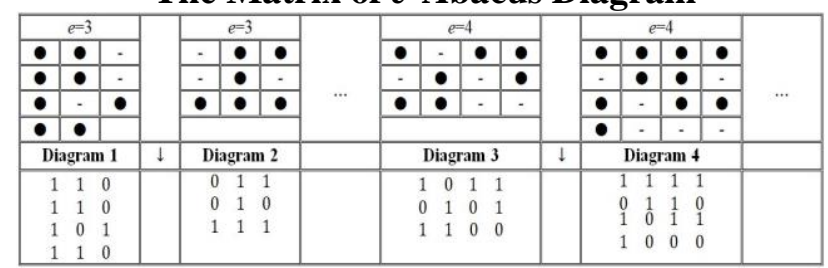

Now, in graph theory, if $a=(u, v)$ is a directed edge of $D, a$ is said to be incident out of $u$ and incident into $v$, also $u$ is said to be adjacent to $v$ and $v$ is adjacent from $u$. The number of directed edges incident out of a vertex $v$ is the outdegreeof $v$ and it's denoted by outdeg $(v)$ and the number of directed edges incident into $v$ is the indegree of $v$ and it's denoted by indeg $(v)$. The sum outdegree and indegree of a vertex $v$ is a degree of $v$, i.e., $\operatorname{deg}(v)=\operatorname{outdeg}(v)+$ indeg .

An directed edge having the same ends is called a loop of $D$ [12]. We can represent the digraph $D$ by matrix which is called adjacent matrix of directed graph and defined as:

$$
m_{i, j}=\left\{\begin{array}{l}
1 ; \text { if }\left(v_{j}, v_{k}\right) \text { is an arc of } D, \\
0 ; \text { otherwise, }
\end{array}\right.
$$

we note that $m_{j, j}=1$ if the $\operatorname{arc}\left(v_{j}, v_{j}\right)$ is a directed loop. Also, from adjacent matrix of digraph can be to obtained the digraph. adjacent matrix of digraph is square matrix, 0 or 1 elements, unconditional symmetric and the sum elements of each row is representation the outdegree of corresponds vertex in row and the sum elements of each column is representation the indegree of corresponds vertex in column. If the digraph without directed loop, then the elements of diagonal matrix are zero.

The following theorem gives the necessary and sufficient condition for an e-Abacus diagram to be an adjacent matrix corresponding to a digraph

Theorem: Every matrix $(0,1) \sim(-, \boldsymbol{\bullet})$ generated from an $e$-abacus diagram is square if and only if it's represents a matrix adjacent to a digraph.

Proof: An $e$-abacus diagram can be represented by a matrix by making each node or slash in the diagram an element with a value of one or zero respectively in the matrix $M$. Suppose that the matrix $M$ of elements $(0,1)$ is a square matrix with size $n \times n$. In order to find an adjacent matrix of $A$ for a digraph corresponding to the matrix resulting from an $e$ Abacus diagram, suppose $\varnothing$ bijective mapping between the row numbers and vertices labels to the indication digraph $D$ of order $n$ has vertex set $V(D)=\left\{v_{1}, v_{2}, \ldots, v_{n}\right\}$, where $\emptyset:\left(Z_{n+1}-\{0\}\right)$ $\rightarrow V(D)$ such that: $\emptyset(j)=v_{j}, j=1,2, \ldots, n$.

Also, in the same method with respect to the column numbers and vertices labels, that is:

$\emptyset(k)=v_{k}, k=1,2, \ldots, n$.

Then $\emptyset(M(j, k))=A\left(v_{j}, v_{k}\right), j, k=1,2, \ldots, n$, where $\varnothing$ is a bijective mapping of the elements of the matrix $M$ to the elements of the adjacent matrix $A$ of a digraph, i.e., $\varnothing: M(j, k) \rightarrow A\left(v_{j}, v_{k}\right), j, k=$ $1,2, \ldots, n . \quad A\left(v_{j}, v_{k}\right)=1$ when $M(j, k)=1$, this means that the vertex $v_{j}$ is adjacent to the vertex $v_{k}$, and $A\left(v_{j}, v_{j}\right)=1$ when $M(j, j)=1$, this means that the vertex is $v_{j}$ incident on a directed loop, as well as $A\left(v_{j}, v_{k}\right)=0$ when $M(j, k)=0$, this means that the vertex $v_{j}$ is not adjacent to the vertex $v_{k}$. So the square matrix $M$ generated from an e-Abacus diagram is adjacent matrix of digraph. Likewise, we can get an e-Abacus diagram from the adjacent matrix of an digraph, this complete the proof .

The matrix $A(3-$ abacus diagram $)=\left[\begin{array}{lll}0 & 1 & 1 \\ 0 & 1 & 0 \\ 1 & 1 & 1\end{array}\right]$ in Diagram 2 is a square matrix, therefore it's represent the adjacent matrix and which corresponding digraph below in Figure 1. 


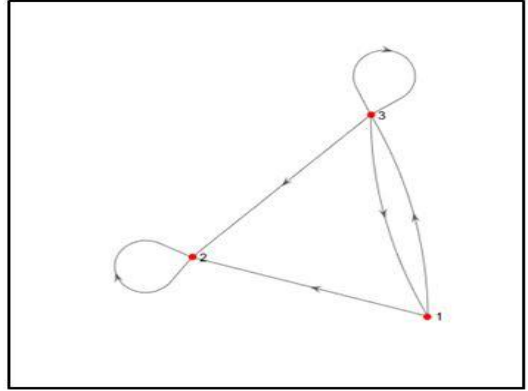

Fig. 1.

\section{Directed Graph of 3 - Abacus Diagram in Diagram 2.}

But the e-abacus diagram representative matrix when $e=3$ in Diagram 1, we can't representation by directed graph because it's not square matrix. If the number of rows in e- Abacus diagram is less than from the number of columns, then we can be obtain on square matrix by addition new rows have elements zeros, this operation does not effected on partition; see Diagram 3, but if the number of columns in e- abacus diagram is less than from the number of rows, then we can be obtain on square matrix by addition new columns have elements zeros, this operation is effected of value of partition, this means choosing $e$ different from the original model, then this case is not taken.

A digraph is symmetric if $(u, v) \in E(D)$, then $(v, u) \in E(D)$. The complete symmetric digraph $K_{n}^{*}$ of order $n$ has both arcs $(u, v)$ and $(v, u)$ for any distinct vertices $u$ and $v$. A digraph is called an oriented graph if $(u, v) \in E(D)$ but $(v, u) \notin E(D)$. Thus, an oriented graph $D$ can be obtained from a graph $G$ by assigning a direction to each edge of $G$ and transforming $G$ itself into an oriented graph. The digraph $D$ is also called an orientation of $G$. An orientation of a complete graph is called a tournament [2].

From the relationship between the of $\mathrm{e}-$ abacus diagram and digraphs, we can get many properties, such as:

1. If the e-abacus diagram representative matrix is identity matrix, then digraph has only directed loop on every vertex.

2. If the e-abacus diagram representative matrix has zero diagonal, then digraph is a simple [2].
3. If the elements of the e-abacus diagram representative matrix $m_{j, k}=1$, for all $j \neq k$, $j, k=1,2, \ldots, n$, then digraph is complete.

4. If the e-abacus diagram representative matrix is upper triangular ( or lower triangular ) matrix, then digraph is not strong [2].

5. If the e-abacus diagram representative matrix is symmetric matrix, then digraph is symmetric.

6. If $m_{j, j+1}=1$ for $j=1,2, \ldots, n-1,(n$ is the number of rows(columns)), then digraph is path digraph [2].

7. If $m_{j, j+1}=1$ for $j=1,2, \ldots, n-1,(n$ is the number rows ( columns)) and $m_{1, n}=1$, then digraph is cycle digraph also it is strong digraph [2].

8. If $m_{j, k}=1$ and $m_{k, j}=0$ for all $j \neq k$ and $j, k=1,2, \ldots, n,(n$ is the number rows ( columns)), then digraph is tournament [1].

Finally, there are another properties can be resulting from the e-Abacus diagram representative matrix.

\section{Application}

Since the e-abacus diagram representative matrix for English letters is square $5 \times 5$, which were presented by Mahmood and Mahmood in [8,9], it can be taken as an application between digraph and partition theory, as the digraph in the encoding formula can be abbreviated as follows: To coding the digraph by 10 -tuple out degrees and indegrees of vertices in D. Code $($ any letter $)=$ (outdeg $v_{1}, \ldots$, outdeg $v_{5} ;$ indeg $v_{1}, \ldots$, indeg $\left.v_{5}\right)$,

where $0 \leq$ outdeg $v_{j} \leq 5$ and $0 \leq$ indeg $v_{j} \leq 5$, for all $1 \leq j \leq 5$. See the appendix 1 .

Perhaps the next question is most important in this area, what will we gain after we have done this step for each letter? The answer will be, if we have any word, how will we be able to convert it according to what has been proposed here!! It is quite clear that we have a square matrix with 5 for the first letter and the same for the second letter and so on for the last letter, let it be $\vartheta$ letters from the chosen word. This means that we now have a new matrix with a capacity of 5 rows and $5 \vartheta$ of columns. Now, added $5(\vartheta-1)$ rows in down the original 
matrix will be become square matrix withsize $5 \vartheta \times$ $5 \vartheta$, all added rows have the number 0 only. For examples:

The word TO:

\section{Adjacent matrix of word TO}

\begin{tabular}{|lllll|lllll|}
\hline \multicolumn{10}{c|}{ T } \\
\hline & & T & & & \multicolumn{1}{c|}{ O } & \\
\hline 0 & 1 & 1 & 1 & 1 & 0 & 1 & 1 & 1 & 0 \\
1 & 0 & 1 & 0 & 0 & 1 & 0 & 0 & 0 & 1 \\
0 & 0 & 1 & 0 & 0 & 1 & 0 & 0 & 0 & 1 \\
0 & 0 & 1 & 0 & 0 & 1 & 0 & 0 & 0 & 1 \\
0 & 0 & 1 & 0 & 0 & 0 & 1 & 1 & 1 & 0 \\
\hline 0 & 0 & 0 & 0 & 0 & 0 & 0 & 0 & 0 & 0 \\
0 & 0 & 0 & 0 & 0 & 0 & 0 & 0 & 0 & 0 \\
0 & 0 & 0 & 0 & 0 & 0 & 0 & 0 & 0 & 0 \\
0 & 0 & 0 & 0 & 0 & 0 & 0 & 0 & 0 & 0 \\
0 & 0 & 0 & 0 & 0 & 0 & 0 & 0 & 0 & 0 \\
\hline
\end{tabular}

Then this word by graph theory, we obtained the digraph in Fig.2 is:

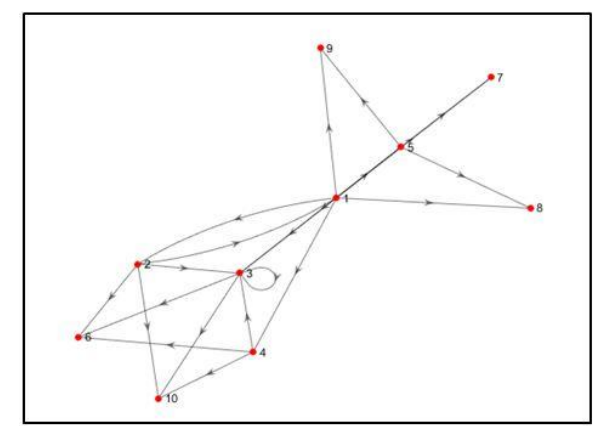

Fig.2.

The digraph from representation the word TO.

The code of the word of $h$ letters is

Code $($ any word $)=\left(\right.$ outdeg $_{1}, \ldots$, outdeg $v_{5}, 0$, outdeg $v_{6}, \ldots$, outdeg $v_{10}, 0, \ldots, 0$, outdeg $v_{h-4}, \ldots$, outdeg $v_{h} ;$ indeg $v_{1}, \ldots$, indeg $_{5}, 0$, inde g $v_{6}, \ldots$, indeg $v_{10}, 0, \ldots, 0$, indeg $v_{h-4}, \ldots$, indeg $\left.v_{h}\right)$.

Hence the code of the word To is:

$(4,2,1,1,1,0,3,2,2,2,3 ; 1,1,5,1,1,3,2,2,2,3)$.

The word BANK:

\section{Adjacent matrix of word BANK}

\begin{tabular}{|c|c|c|c|c|c|c|c|c|c|c|c|c|c|c|c|c|c|c|c|}
\hline \multicolumn{20}{|c|}{ BANK } \\
\hline \multicolumn{5}{|c|}{ B } & \multicolumn{5}{|c|}{$\mathrm{A}$} & \multicolumn{5}{|c|}{$\mathrm{N}$} & \multicolumn{5}{|c|}{$\mathbf{K}$} \\
\hline 0 & & 1 & 1 & 0 & 0 & 1 & 1 & 1 & 0 & 0 & 1 & 0 & 0 & 1 & 0 & 1 & 0 & 0 & 1 \\
\hline 0 & 1 & 0 & 0 & 1 & 1 & 0 & 0 & 0 & 1 & 0 & 1 & 1 & 0 & 1 & 0 & 1 & 0 & 1 & 0 \\
\hline 0 & 1 & 1 & 1 & 0 & 1 & 1 & 1 & 1 & 1 & 0 & 1 & 1 & 1 & 1 & 0 & 1 & 1 & 0 & 0 \\
\hline 0 & 1 & 0 & 0 & 1 & 1 & 0 & 0 & 0 & 1 & 0 & 1 & 0 & 1 & 1 & 0 & 1 & 0 & 1 & 0 \\
\hline 0 & 1 & 1 & 1 & 0 & 1 & 0 & 0 & 0 & 1 & 0 & 1 & 0 & 0 & 1 & 0 & 1 & 0 & 0 & 1 \\
\hline 0 & 0 & 0 & 0 & 0 & 0 & 0 & 0 & 0 & 0 & 0 & 0 & 0 & 0 & 0 & 0 & 0 & 0 & 0 & 0 \\
\hline 0 & 0 & 0 & 0 & 0 & 0 & 0 & 0 & 0 & 0 & 0 & 0 & 0 & 0 & 0 & 0 & 0 & 0 & 0 & 0 \\
\hline 0 & 0 & 0 & 0 & 0 & 0 & 0 & 0 & 0 & 0 & 0 & 0 & 0 & 0 & 0 & 0 & 0 & 0 & 0 & 0 \\
\hline 0 & 0 & 0 & 0 & 0 & 0 & 0 & 0 & 0 & 0 & 0 & 0 & 0 & 0 & 0 & 0 & 0 & 0 & 0 & 0 \\
\hline 0 & 0 & 0 & 0 & 0 & 0 & 0 & 0 & 0 & 0 & 0 & 0 & 0 & 0 & 0 & 0 & 0 & 0 & 0 & 0 \\
\hline 0 & 0 & 0 & 0 & 0 & 0 & 0 & 0 & 0 & 0 & 0 & 0 & 0 & 0 & 0 & 0 & 0 & 0 & 0 & 0 \\
\hline 0 & 0 & 0 & 0 & 0 & 0 & 0 & 0 & 0 & 0 & 0 & 0 & 0 & 0 & 0 & 0 & 0 & 0 & 0 & 0 \\
\hline 0 & 0 & 0 & 0 & 0 & 0 & 0 & 0 & 0 & 0 & 0 & 0 & 0 & 0 & 0 & 0 & 0 & 0 & 0 & 0 \\
\hline 0 & 0 & 0 & 0 & 0 & 0 & 0 & 0 & 0 & 0 & 0 & 0 & 0 & 0 & 0 & 0 & 0 & 0 & 0 & 0 \\
\hline 0 & 0 & 0 & 0 & 0 & 0 & 0 & 0 & 0 & 0 & 0 & 0 & 0 & 0 & 0 & 0 & 0 & 0 & 0 & 0 \\
\hline 0 & 0 & 0 & 0 & 0 & 0 & 0 & 0 & 0 & 0 & 0 & 0 & 0 & 0 & 0 & 0 & 0 & 0 & 0 & 0 \\
\hline 0 & 0 & 0 & 0 & 0 & 0 & 0 & 0 & 0 & 0 & 0 & 0 & 0 & 0 & 0 & 0 & 0 & 0 & 0 & 0 \\
\hline 0 & 0 & 0 & 0 & 0 & 0 & 0 & 0 & 0 & 0 & 0 & 0 & 0 & 0 & 0 & 0 & 0 & 0 & 0 & 0 \\
\hline 0 & 0 & 0 & 0 & 0 & 0 & 0 & 0 & 0 & 0 & 0 & 0 & 0 & 0 & 0 & & 0 & 0 & 0 & 0 \\
\hline
\end{tabular}

The digraph of the word BANK is:

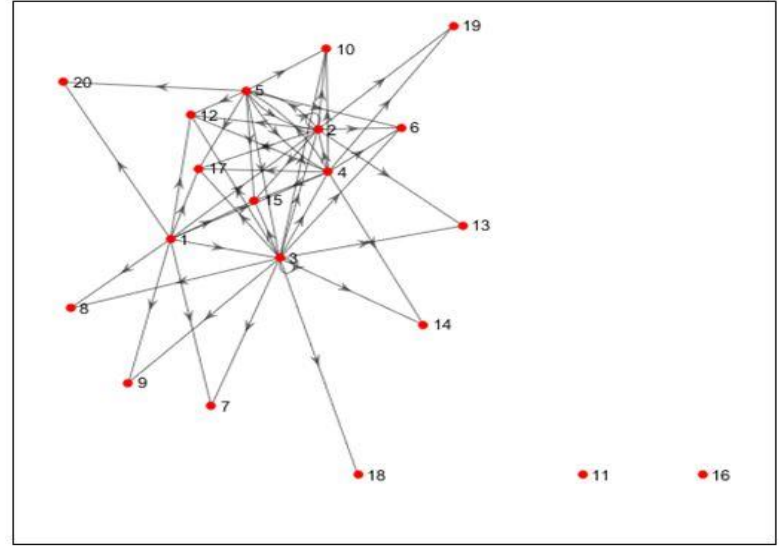

Fig.3.

The digraph from representation the word BANK.

and the code is :

$(3,2,3,2,3,0,3,2,5,2,2,02,3,4,3,2,0,2,2,2,2,2$;

$0,5,3,3,2,0,4,2,2,2,4,0,0,5,2,2,5,0,0,5,1,2,2)$.

\section{Conclusion}

1. Any partition can be represented according to four equivalent stages (Young tableau, $e$ abacus diagram adjacent matrix and by graph theory).

2. In order to achieve the first step, the matrix chosen must be square in order for us to represent it in graph theory.

3. A smooth transition to some cases and characteristics of graph theory, and in other cases we need additional conditions in order to reach those basic rules for cases of graph theory.

4. The possibility of creating code for any matrix of $e$-abacus diagram.

5. Change any word and make it graph.

6. In the case of the sentence, the following mechanism is:

Assume that the number of sentence burns (not counting the spaces between the words) is $a$ and that the number of spaces between one word and another is $b$ and therefore the square matrix that will be produced for this sentence will have a capacity of $5(a+b)$, and this means that the number of graph vertices generated will be $5(a+b)$

7. It is possible to use this new technology in special concepts related to some $e$-abacus diagram applications, see [7]. 
Acknowledgements:

The authors sincerely thank the University of Mosul/College of Education for Pure Sciences and the College of Computer Science and Mathematics for moral support in order to complete this research.

\section{References:}

[1] R. Balakrishnan and K. Ranganathan, A Textbook of Graph Theory, Springer Science, New York 2012.

[2] G. Chartrand and L. Lesniak, Graphs and Digraphs, $6^{\text {th }}$ ed., Wadsworth and Brooks / Cole. California, 2016.

[3] J.C. Fournier, Graph Theory and Applications, Library of Congress Cataloging-inPublication Data, 2009.

[4] W. Fulton, Young Tableaux, with Applications to Representation Theory and Geometry, Cambridge University Press, 1997.

[5] G. D. James, Some combinatorial results involving Young diagrams, Mathematical Proceeding of the Cambridge Philosophical Society, Vol.83,1978,pp. 1-10.

[6] L. G. Jonathan, Y. Jay and A. Mark, Graph Theory and Its Applications, $3^{\text {rd }}$ edition. LLC CRC Press is an imprint of Taylor \& Francis Group, 2019.

[7] A. S. Mahmood, Replace the content in eabacus diagram. Open Access Library J., Vol.7,2020, pp.1-6.

[8] A. B. Mahmood and A. S. Mahmood, Secretword by e-abacus diagram I. Iraqi J. of Science, Vol.60, No.3, 2019, pp.638-646.

[9] A. B. Mahmood and A. S. Mahmood, Secrettext by e-abacus diagram II. Iraqi J. of Science, Vol.60, No.4,2019, pp.840--846.

[10] A. Mathas, Iwahori-Hecke Algebras and Schur Algebras of the Symmetric Group, University Lecture Series, Vol.15, 1999.

[11] D. Matthias and E. Frank, Quantitative Graph Theory Mathematical Foundations and Applications, Chapman and Hall/CRC. 2019.

[12] J. W. Robin, Introduction to Graph Theory, $4^{\text {th }}$ edition, Library of Congress Cataloging in Publication Data, 1996.

\section{Contribution of individual authors to the creation of a scientific article (ghostwriting policy)}

Ahmed Mohammed Ali was study the directed graph, some properties and sketch the digraphs.

Ammar Seddik Mahmmod give idea partition theory with connection to digraph.

\section{Sources of funding for research presented in a scientific article or scientific article itself}

There are no sources of funding

\section{Creative Commons Attribution}

\section{License 4.0 (Attribution 4.0 \\ International , CC BY 4.0)}

This article is published under the terms of the Creative Commons Attribution License 4.0 https://creativecommons.org/licenses/by/4.0/deed.en_US 


\section{See the appendix}

\section{The digraphs of English letters}

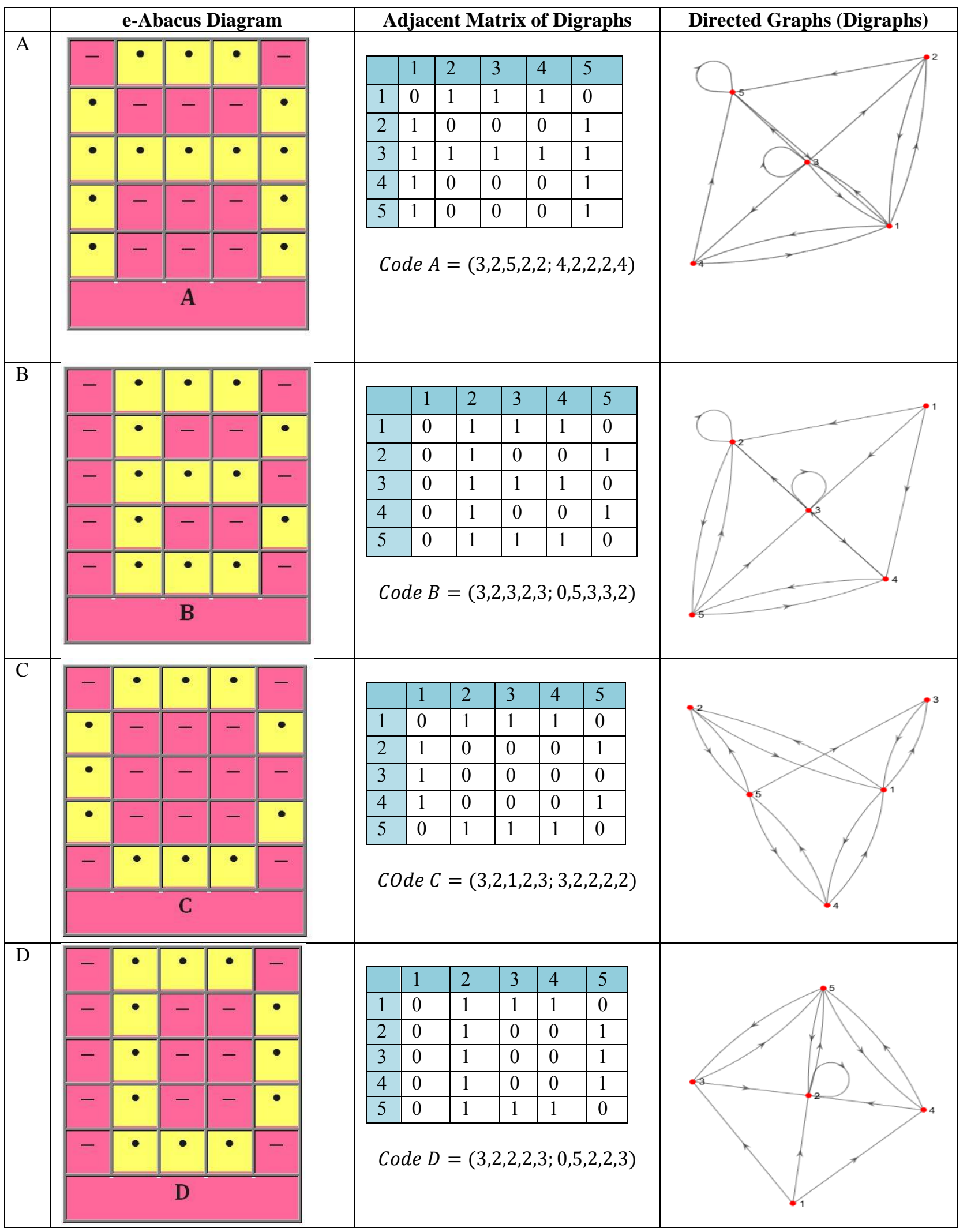




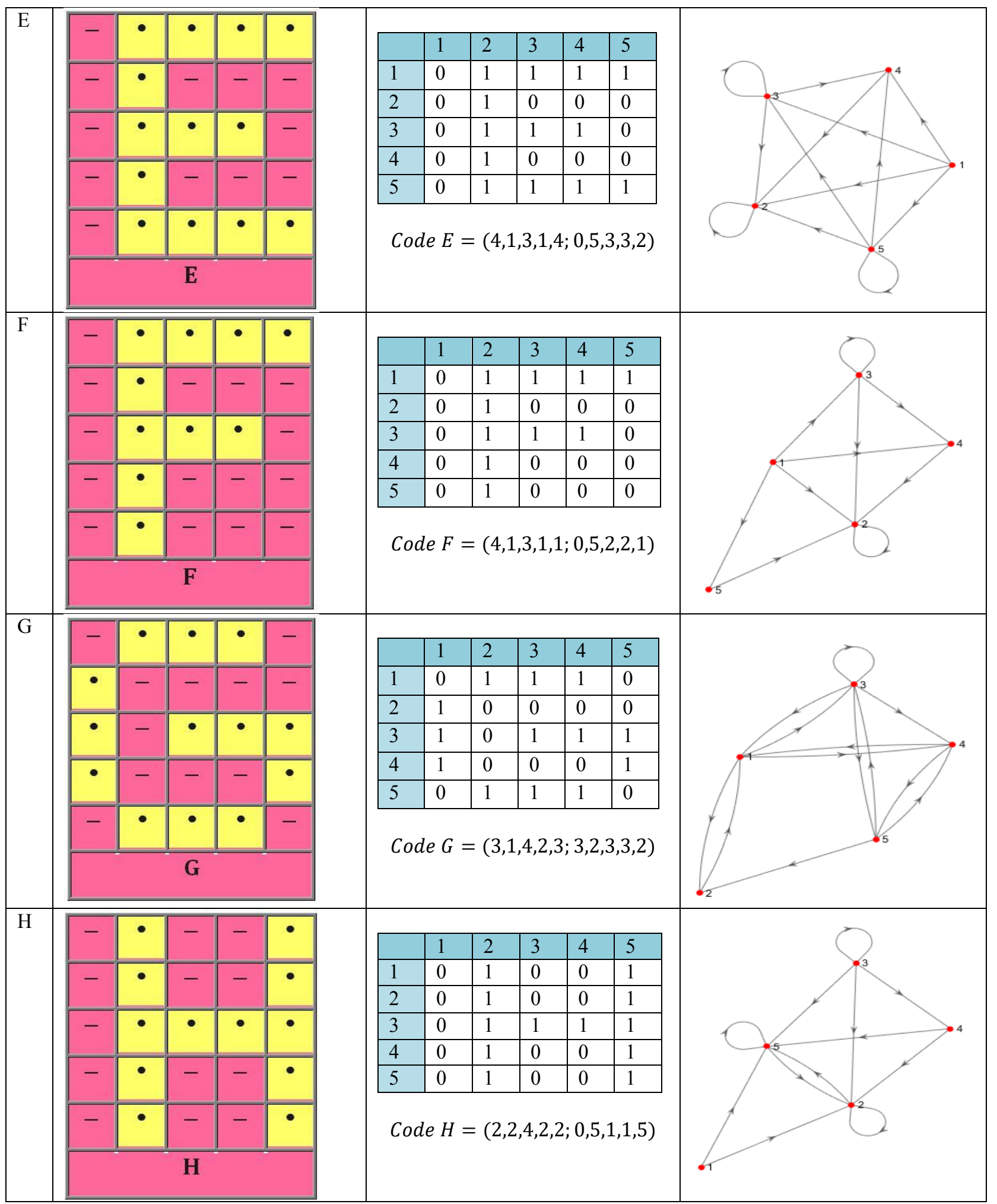




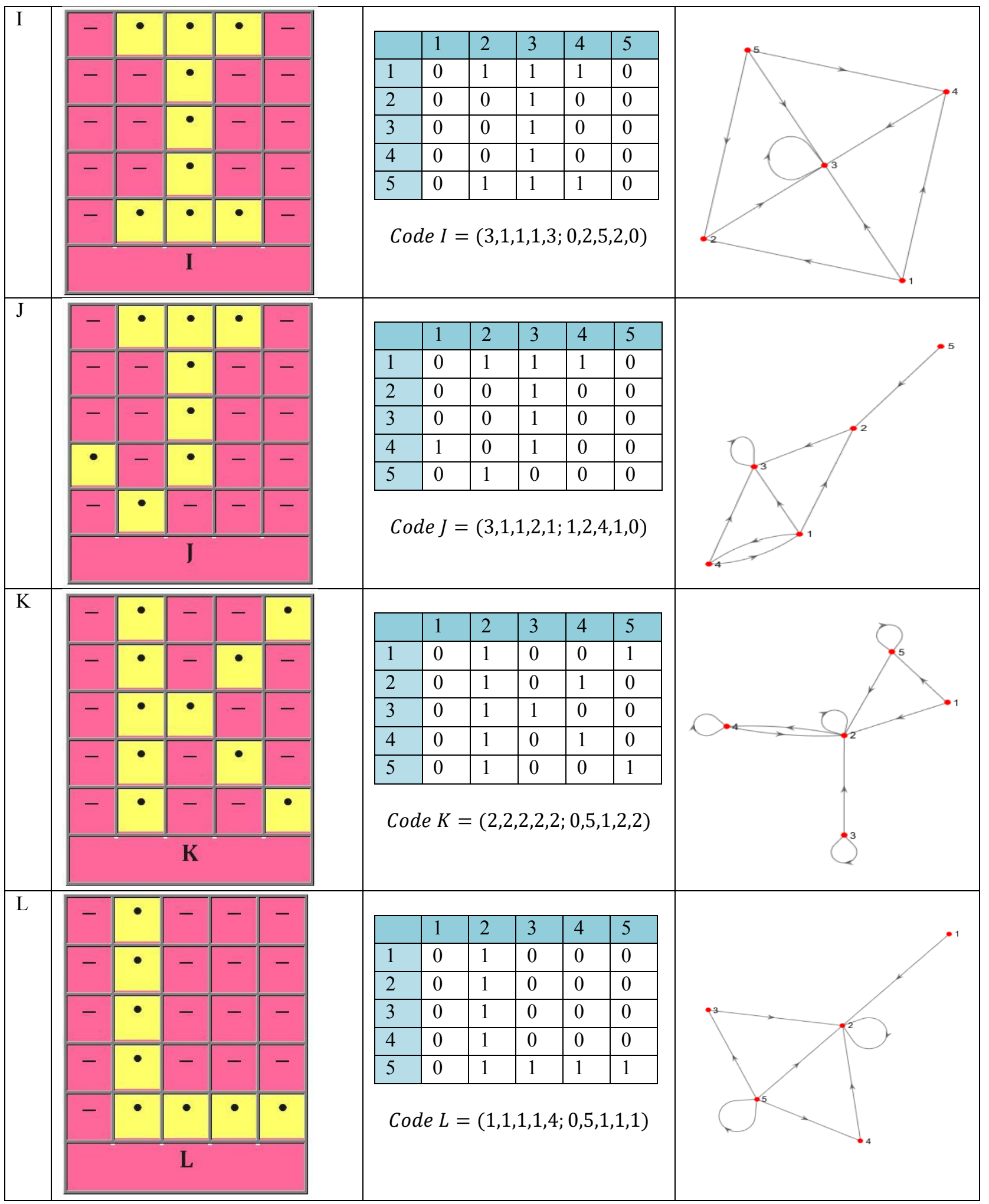




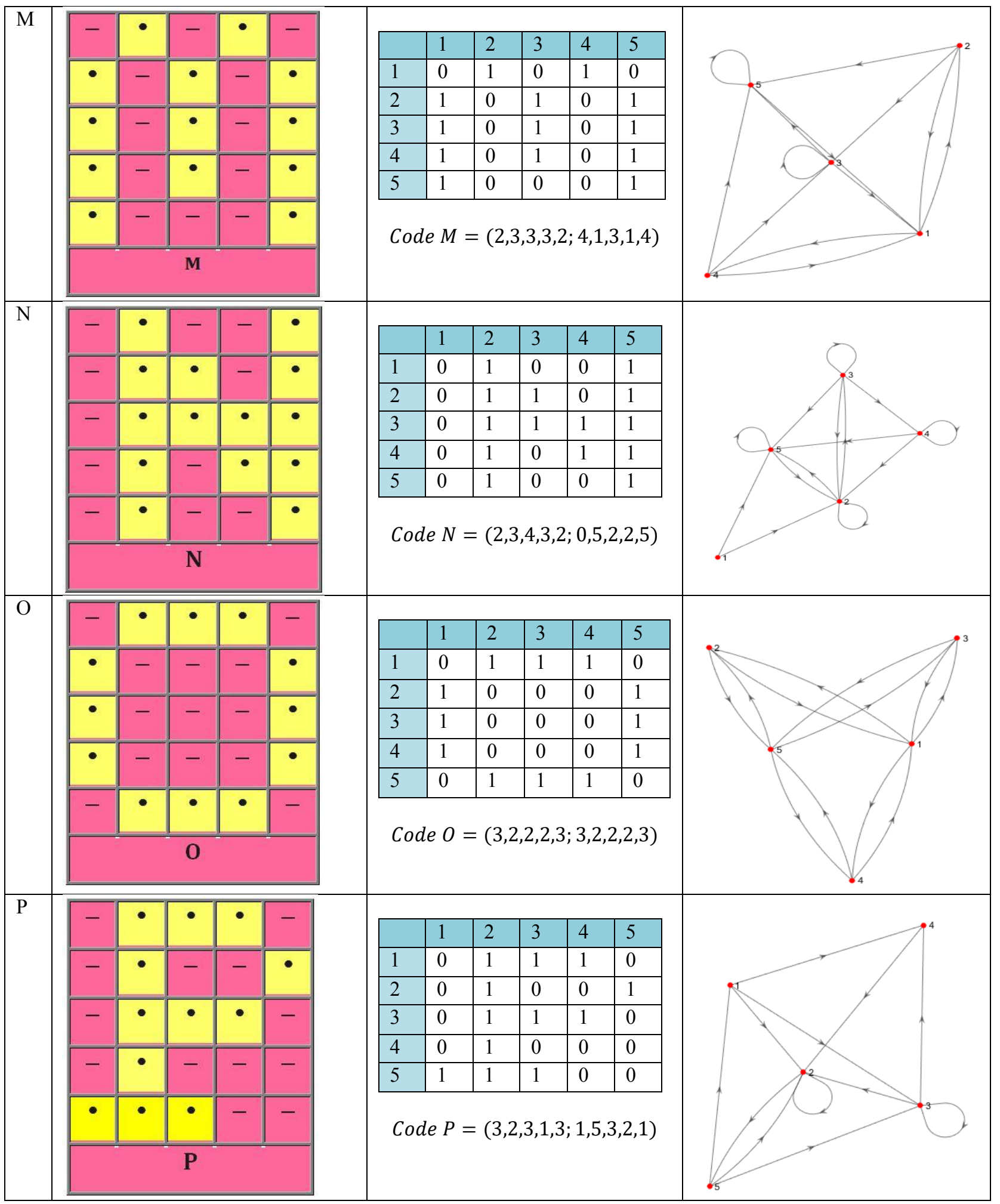




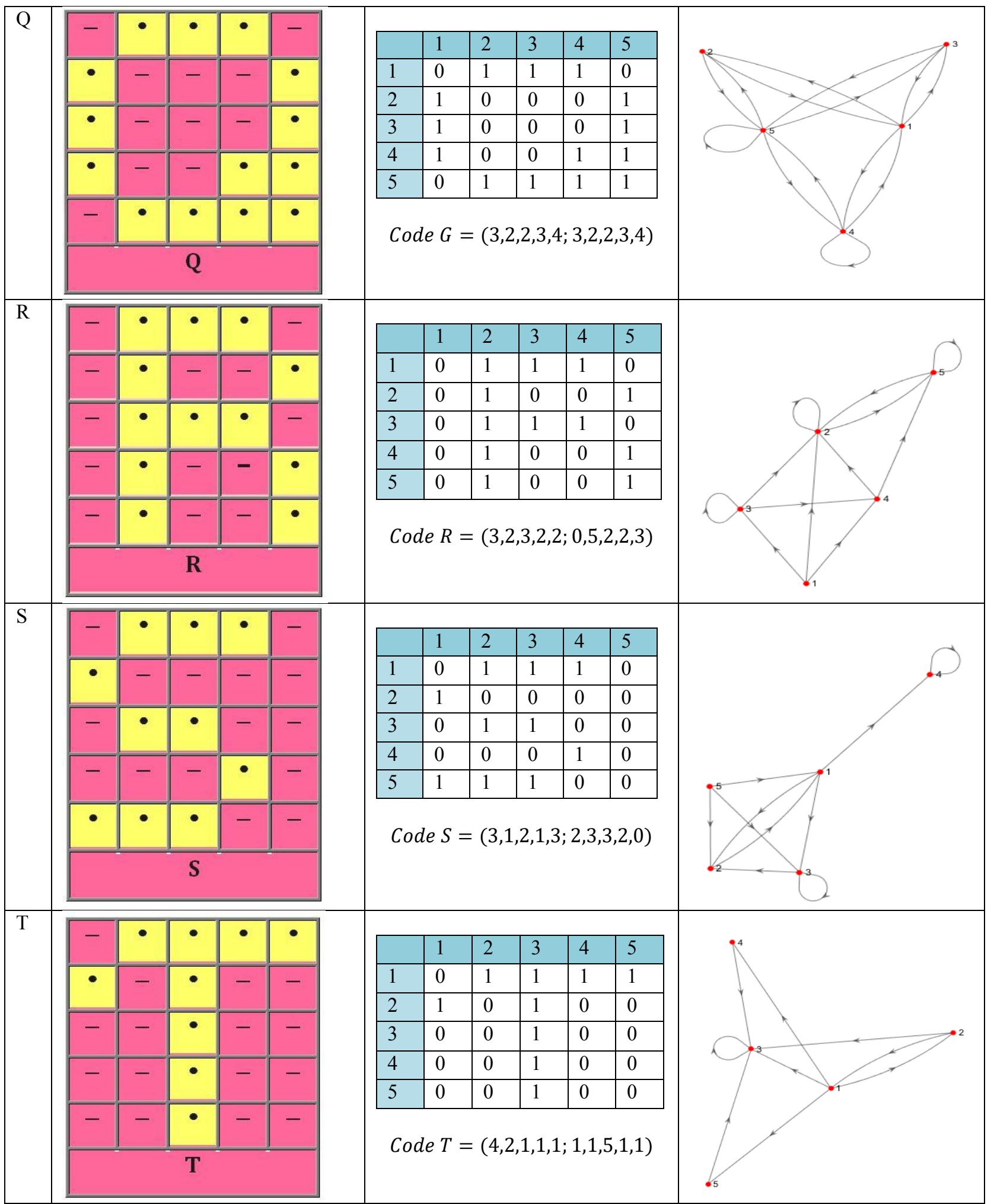




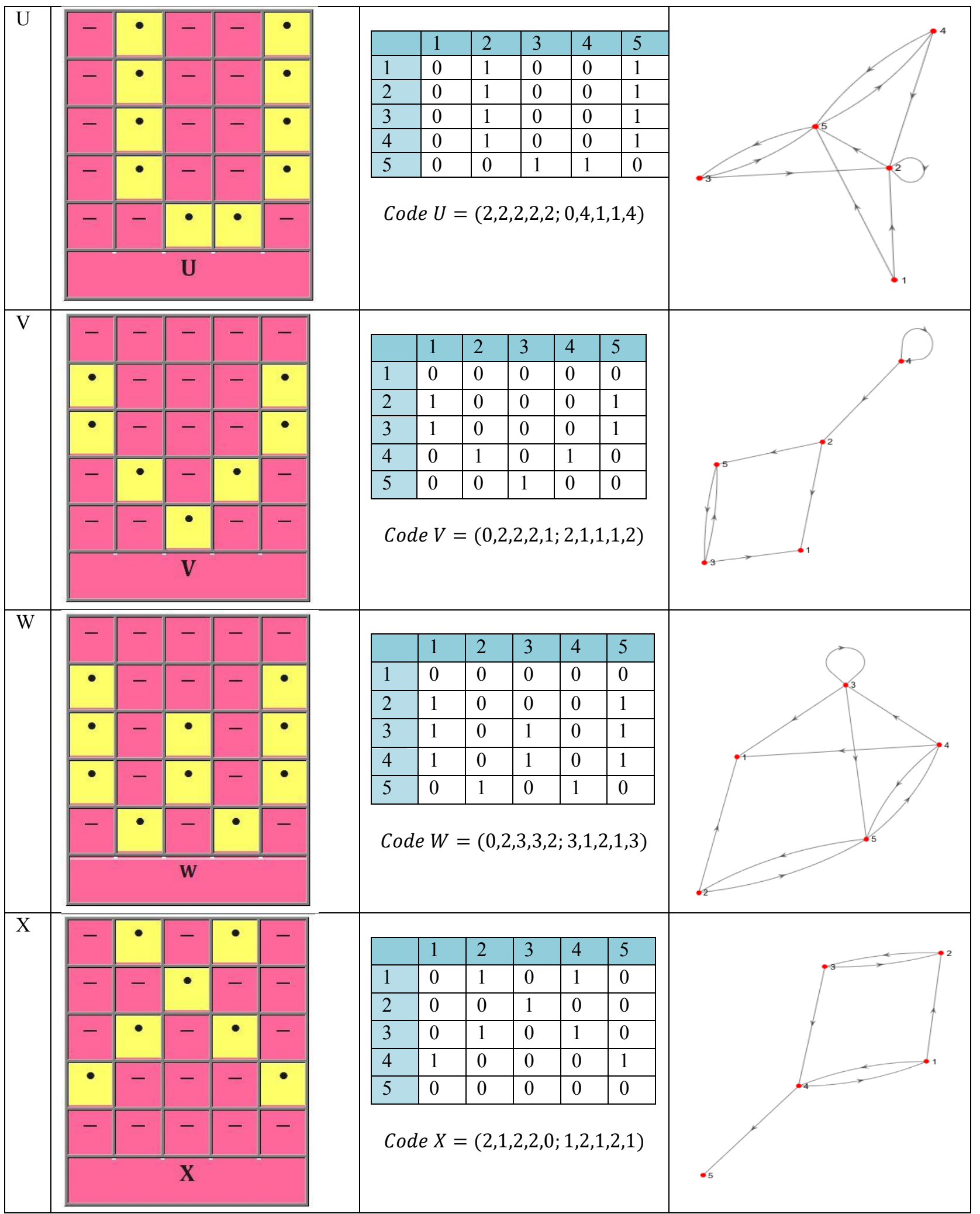




\begin{tabular}{|c|c|c|c|c|c|c|c|c|c|c|c|c|}
\hline \multirow[t]{9}{*}{$\mathrm{Y}$} & \multirow[t]{2}{*}{-} & \multirow{2}{*}{-} & \multirow{2}{*}{-} & \multirow{2}{*}{-} & \multirow{2}{*}{-} & & & & & & \multirow[b]{2}{*}{5} & \multirow{9}{*}{$\int^{4}$} \\
\hline & & & & & & & 1 & 2 & 3 & 4 & & \\
\hline & $\bullet$ & - & - & - & $\bullet$ & 1 & 0 & 0 & 0 & 0 & 0 & \\
\hline & & & & & & 2 & 1 & 0 & 0 & 0 & 1 & \\
\hline & - & $\bullet$ & $\bullet$ & $\bullet$ & - & 3 & 0 & 1 & 1 & 1 & 0 & \\
\hline & - & - & $\bullet$ & - & - & 4 & 0 & 0 & 1 & 0 & 0 & \\
\hline & & & & & & 5 & 0 & 0 & 1 & 0 & 0 & \\
\hline & - & - & $\bullet$ & - & - & \multirow{2}{*}{\multicolumn{6}{|c|}{ Code $Y=(0,2,3,1,1 ; 1,1,3,1,1)$}} & \\
\hline & \multicolumn{5}{|c|}{$\mathbf{Y}$} & & & & & & & \\
\hline \multirow[t]{9}{*}{$\mathrm{Z}$} & \multirow{2}{*}{-} & \multirow{2}{*}{$\bullet$} & \multirow{2}{*}{$\bullet$} & \multirow{2}{*}{$\bullet$} & \multirow[t]{2}{*}{ • } & & & & & & & \multirow{9}{*}{ 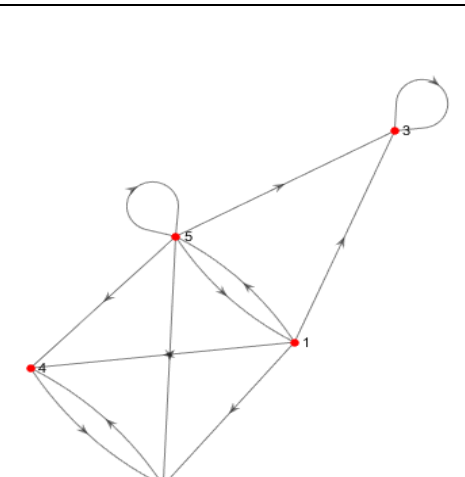 } \\
\hline & & & & & & & 1 & 2 & 3 & 4 & 5 & \\
\hline & - & - & - & $\bullet$ & - & 1 & 0 & 1 & 1 & 1 & 1 & \\
\hline & & & & & & 2 & 0 & 0 & 0 & 1 & 0 & \\
\hline & - & - & $\bullet$ & - & - & 3 & 0 & 0 & 1 & 0 & 0 & \\
\hline & & & & & & 4 & 0 & 1 & 0 & 0 & 0 & \\
\hline & - & $\bullet$ & - & - & - & 5 & 1 & 1 & 1 & 1 & 1 & \\
\hline & • & $\bullet$ & $\bullet$ & $\bullet$ & $\bullet$ & \multirow{2}{*}{\multicolumn{6}{|c|}{ Code $Z=(4,1,1,1,5 ; 1,3,3,3,2)$}} & \\
\hline & \multicolumn{5}{|c|}{$\mathbf{Z}$} & & & & & & & \\
\hline
\end{tabular}

